\title{
The effects of kangaroo mother care on the time to breastfeeding initiation among preterm and LBW infants: a meta-analysis of published studies
}

\author{
Alemayehu Gonie Mekonnen *ib, Sisay Shewasinad Yehualashet and Alebachew Demelash Bayleyegn
}

\begin{abstract}
Background: Kangaroo mother care is a comprehensive intervention given for all newborns especially for premature and low birthweight infants. It is the most feasible and preferred intervention for decreasing neonatal morbidity and mortality. Even though time to initiating breastfeeding has been examined by randomized controlled trials, varying findings have been reported. Therefore, the main objective of this meta-analysis was to estimate the pooled mean time to initiate breastfeeding among preterm and low birthweight infants.
\end{abstract}

Methods: The authors searched for randomized controlled trial studies conducted on the effects of kangaroo mother care on the time to breastfeeding initiation among preterm and low birthweight infants. Published articles were identified through a computerized search of electronic databases that includes MEDLINE via PubMed, EMBASE, CINAHL and CENTRAL. The search terms were kangaroo mother care or (skin to skin), or conventional care, newborns, preterm infants, low birthweight infants and randomized controlled trial. A total of 467 eligible titles were identified and eight studies met the inclusion criteria. The extracted data were entered and analyzed using Cochrane Review Manager-5-3 software. Heterogeneity across studies was evaluated by $\mathrm{Chi}^{2}$ test and inconsistency index $\left(\mathrm{I}^{2}\right)$. Publication bias was assessed using a funnel plot. The random effect model was applied to estimate the pooled mean time to initiate breastfeeding with 95\% confidence interval.

Results: In this meta-analysis, the overall pooled mean time to initiate breastfeeding was 2.6 days $(95 \% \mathrm{Cl} 1.23,3.96)$. Preterm and low birthweight infants receiving kangaroo mother care intervention initiated breastfeeding 2 days $14 \mathrm{~h}$ 24 min earlier than conventional care of radiant warmer/incubator method.

Conclusions: Kangaroo mother care promotes early initiation of breastfeeding as compared to conventional care method. Therefore, health facilities need to implement the kangaroo mother care for preterm and low birthweight infants.

Keywords: Kangaroo mother care, Initiation of breastfeeding, Preterm and LBW infants

\footnotetext{
* Correspondence: alemayehugonie19@gmail.com

Department of Nursing, College of Health Sciences, Debre Berhan University,
}

Debre Berhan, Ethiopia

(c) The Author(s). 2019 Open Access This article is distributed under the terms of the Creative Commons Attribution 4.0 International License (http://creativecommons.org/licenses/by/4.0/) which permits unrestricted use, distribution, and reproduction in any medium, provided you give appropriate credit to the original author(s) and the source, provide a link to the Creative Commons license, and indicate if changes were made. The Creative Commons Public Domain Dedication waiver (http://creativecommons.org/publicdomain/zero/1.0/) applies to the data made available in this article, unless otherwise stated. 


\section{Background}

Kangaroo mother care (KMC) was first started in Colombia in 1978 [1]. It is a comprehensive intervention given for all newborns especially for premature and low birthweight (LBW) infants. Kangaroo mother care is the most feasible, readily available, and preferred intervention for decreasing neonatal morbidity and mortality in developed and developing countries, and suitable for use in all settings [2]. It is based on three components: kangaroo position (skin-to-skin contact between mother and infant), breastfeeding; and timely discharge with close follow-up [3]. This procedure could be done at any time during the night and day [4] and creates an optimal environment for adaptation of newborn infants to extra-uterine life [5].

Skin-to-skin contact between the mother and the baby is a safe and inexpensive procedure that has proven benefits for mothers and children as compared to an incubator caring method. It plays a significant role on infant survival, neurodevelopment, and the quality of mother-infant bonding. Kangaroo mother care complements good quality care and allows providers to ration use of expensive resources such as warmers and incubators $[6,7]$.

In preterm and LBW infants, skin-to-skin contact between the mother and her infant decreases maternal postpartum depressive symptoms [8] and improves self-efficacy and mother-child bonding [9]. Preterm and LBW infants who are receiving KMC gain more weight per day, have better heart rate and breathing regulation, and have effective oxygenation preterm $[4,10]$. Furthermore, it facilitates the newborn early initiation and effective breastfeeding [11], and in turn effective breastfeeding reduces the incidence of necrotizing enterocolitis which is a leading cause of death in preterm infants [12, 13].

Kangaroo mother care for preterm infants is also related to better cognitive and motor development at six months of age [8]. Other than the neonatal and maternal health benefits, KMC is an important tool in reducing the postpartum hospital stay, thereby cutting down the overall healthcare expenditure and provides economic benefit to the parents [7]. Preterm and LBW infants who were given KMC spent less time in the hospital than those who were given standard care [14]. Even though time to initiating breastfeeding was examined by randomized control trial studies, varying findings were stated. Therefore, the main objective of this meta-analysis was to estimate the pooled mean time to initiate breastfeeding among preterm and low birthweight infants.

\section{Hypothesis}

There is a mean time difference between kangaroo mother care and the conventional care method to initiate breastfeeding among preterm and LBW infants.
Preterm and LBW infants receiving KMC have earlier initiation of breastfeeding than conventional care (radiant warmer/incubator).

\section{Methods}

This review covered all randomized controlled trial studies of kangaroo mother care versus conventional care method and its breastfeeding initiation among premature and LBW infants. The search strategy focused on human's category and included randomized controlled trial studies published from January 2000 to June 30, 2018. The review authors searched the studies using the following databases: MEDLINE via PubMed, EMBASE, CINAHL (Cumulative Index to Nursing and Allied Health Literature) and CENTRAL (Cochrane Central Register of Controlled Trials). The search terms were KMC or (skin to skin), or conventional care method, newborns, preterm infants, LBW infants, randomized controlled trial (Additional file 1). These key terms were combined using Boolean operators "AND" and "OR" to narrow the search. In addition, we searched reference lists of identified studies, peer reviewed articles, and Google Scholar to augment database searches. To identify relevant articles, titles and abstracts of retrieved articles were exported to Endnote to screen duplicate articles. Then three review authors assessed and reviewed independently all studies deemed suitable to determine inclusion.

\section{Study selection criteria}

- Inclusion criteria: To meet inclusion criteria, titles and abstracts of studies were examined, and the following inclusion criteria were included

- Full text randomized controlled trial study design

- Studies published after year 2000 (to minimize the time lag bias)

- Peer-reviewed and published in the English language

- Reported the mean time and standard deviations to initiate exclusive breastfeeding

- Exclusion criteria: The following exclusion criteria were used in screening articles for the current metaanalysis:

- Reported the knowledge and practice of mothers towards KMC

- Studies that repeated findings from the already included studies

- Studies of KMC on the normal infants

\section{Study selection and data extraction}

The authors screened all identified titles and abstracts. Articles found relevant by title and abstract were undergone for full-text review for eligibility. For eligible studies, three 
Table 1 Summary of RCT studies included in the analysis, June 30, 2018

\begin{tabular}{|c|c|c|c|c|c|c|c|c|c|c|c|}
\hline \multirow[t]{2}{*}{ Sr. } & \multirow[t]{2}{*}{ Author } & \multirow[t]{2}{*}{ Publication year } & \multicolumn{3}{|c|}{$\begin{array}{l}\text { KMC mean time to initiate } \\
\text { breastfeeding }\end{array}$} & \multicolumn{3}{|c|}{ Radiator warmer } & \multirow[t]{2}{*}{ Study design } & \multirow[t]{2}{*}{ Inclusion criteria } & \multirow[t]{2}{*}{ Setting } \\
\hline & & & mean & SD & Total & mean & SD & Total & & & \\
\hline 1 & Rahman et al. [18] & 2017 & 9.10 & 2.40 & 40 & 14.70 & 4.50 & 40 & $\mathrm{RCT}$ & Preterm + LBW & Bangladesh \\
\hline 2 & Mahbubul et al. [17] & 2017 & 9.53 & 3.35 & 40 & 14.35 & 6.06 & 40 & $\mathrm{RCT}$ & LBW & Bangladesł \\
\hline 3 & Lamy Filho et al. [16] & 2008 & 18.90 & 11.4 & 354 & 24.10 & 12.8 & 609 & $\mathrm{RCT}$ & LBW & Brazil \\
\hline 4 & Sharma et al. [20] & 2016 & 10.10 & 5.90 & 71 & 10.80 & 4.30 & 70 & $\mathrm{RCT}$ & Preterm + LBW & India \\
\hline 5 & Suman Rao et al. [22] & 2008 & 5.71 & 5.65 & 103 & 4.85 & 4.94 & 103 & $\mathrm{RCT}$ & LBW & India \\
\hline 6 & Ghavane et al. [21] & 2012 & 9.70 & 6.40 & 71 & 11.00 & 8.10 & 69 & $\mathrm{RCT}$ & LBW & India \\
\hline 7 & Jagadale \& Salunkhe [15] & 2014 & 5.4 & 1.10 & 25 & 6.7 & 2.92 & 25 & $\mathrm{RCT}$ & LBW & India \\
\hline 8 & Srivastava et al. [19] & 2014 & 6.71 & 1.89 & 118 & 9.55 & 1.14 & 122 & $\mathrm{RCT}$ & Preterm & India \\
\hline
\end{tabular}

authors extracted the data using the pre-determined inclusion criteria. The review authors recorded the data on Microsoft excel and extracted the following data for each trial: the authors, year of publication, study setting, study design, sample size, and inclusion criteria (Table 1). The study selection and data extraction were done from April 10 to June 30, 2018. Data extraction was performed independently by three review authors (AG, SS and AD) using a pre-specified data extraction form. Discrepancies were solved through discussion and articles were included after consensus was reached. The authors communicated the trial authors for clarification.

\section{Data analysis}

The extracted data were entered and analyzed using a Cochrane review manager-5-3 software. Potential sources of heterogeneity across studies was evaluated by $\mathrm{Chi}^{2}$ test which verifies the presence of heterogeneity, and inconsistency index $\left(\mathrm{I}^{2}\right)$ which describes the percentage of total variation across studies. The $\mathrm{I}^{2}$ provides the percentage of variability due to heterogeneity rather than the chance difference or sampling error. The $\mathrm{I}^{2}$ greater than $75 \%$ and $\mathrm{Chi}^{2}$ test $(p<0.10)$ was considered statistically significant heterogeneity [13]. The random effects model which assesses the variability within and between studies was applied to estimate the pooled mean time to initiate exclusive breastfeeding with 95\% confidence interval.

Publication bias was assessed using the funnel plot (which displays effect sizes plotted against the sample size, standard error, conditional variance, or some other measure of the precision of the estimate). In the presence of a cloud of data points that is symmetric around the population effect size and has the shape of a funnel, one can conclude as no publication bias [13]. Sensitivity analysis was performed

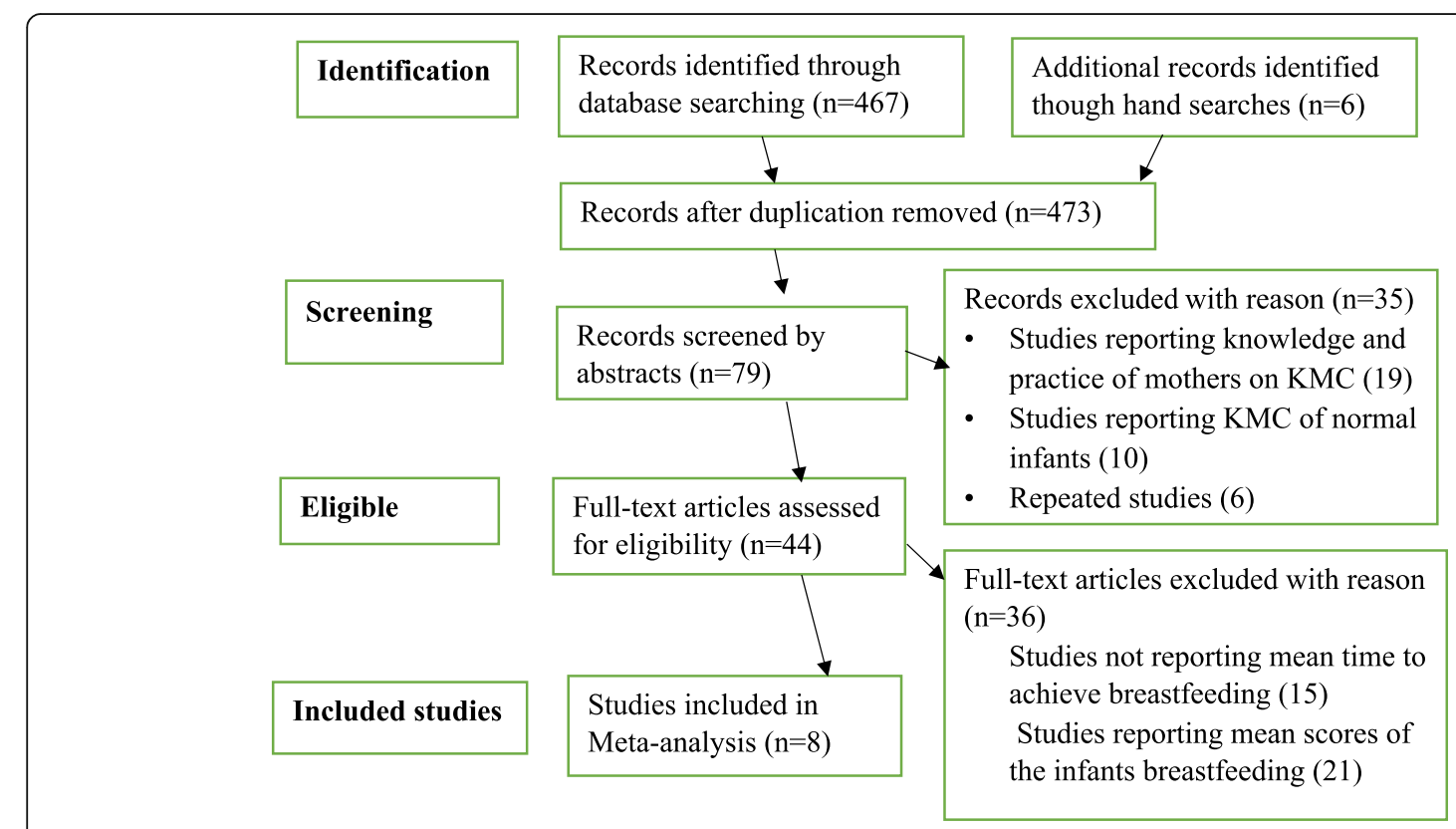

Fig. 1 PRISMA flow-diagram that depicts the phases of study selection 


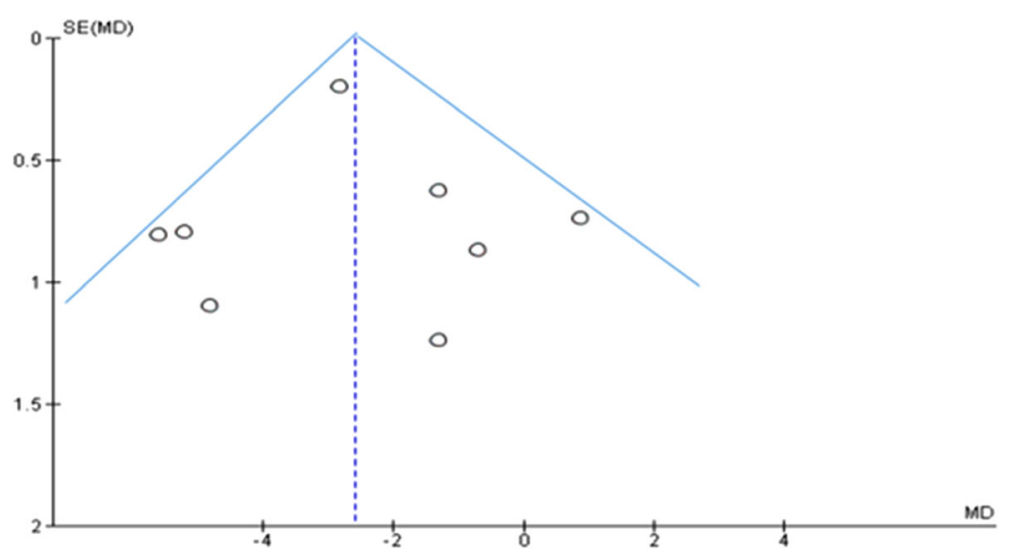

Fig. 2 Funnel plot showing publication bias (inverted symmetrical funnel plot)

to explore the effects of random-effects analyses for primary outcomes with statistical heterogeneity. In addition, to be free of other sources of bias, review authors independently assessed risk of bias in included studies and resolved discrepancies through discussion.

\section{Results}

\section{Characteristics of included studies}

Totally, 467 studies were retrieved, of which 44 were identified as being potentially eligible for this review. Altogether, eight randomized control trial studies (Table 1) comprising of 1900 participants were included in the meta-analysis (Fig. 1). All included articles were facility-based studies and conducted in neonatal care and postnatal care units. The studies included preterm and LBW neonates with a sample size ranging from 50 [15] to 963 [16]. Among the articles included in the analysis, seven studies reported that neonate in the KMC group initiated exclusive breastfeeding earlier than the conventional care neonates [15-21]. On the contrary, one study reported that conventional care neonate initiated breastfeeding earlier than KMC neonates [22]. The studies were conducted in India [15, 19-22], Brazil [16], Bangladesh [17, 18].

\section{Heterogeneity and publication bias}

The included studies were assessed for heterogeneity and publication bias. Accordingly, the analysis showed a substantial heterogeneity among studies with $\mathrm{Chi}^{2}=$ 61.78; $p<0.00001$ and $\mathrm{I}^{2}$ statistics $\left(\mathrm{I}^{2}=89 \%\right)$. The publication bias was checked by a funnel plot, and the plot has a symmetric inverted funnel shape showing no evidence of variability in effect sizes from studies and publication bias (Fig. 2).

\section{Sensitivity analysis}

Sensitivity analysis of the eight studies were performed to test the effect of each study on the pooled result and no statistical source of heterogeneity among the eight studies were identified.

\section{The pooled mean time to initiate breastfeeding}

The pooled mean time to initiate breastfeeding is presented in a forest plot (Fig. 3). The reported mean time to initiate breastfeeding in each study ranged from around half day [20] to 5 days [18] with a substantial heterogeneity across studies $\left(\mathrm{Chi}^{2}=61.78 ; p<0.00001\right.$; $\left.\mathrm{I}^{2}=89 \%\right)$. In this meta-analysis, the overall pooled mean time for initiating breastfeeding from the random effects model revealed 2.6 days ( 2 days $14 \mathrm{~h} 24 \mathrm{~min}$ ) (95\% CI $1.23,3.96)$. This indicated that preterm and low birthweight infants in the KMC intervention group initiated exclusive breastfeeding 2.6 days earlier than conventional care method (Fig. 3).

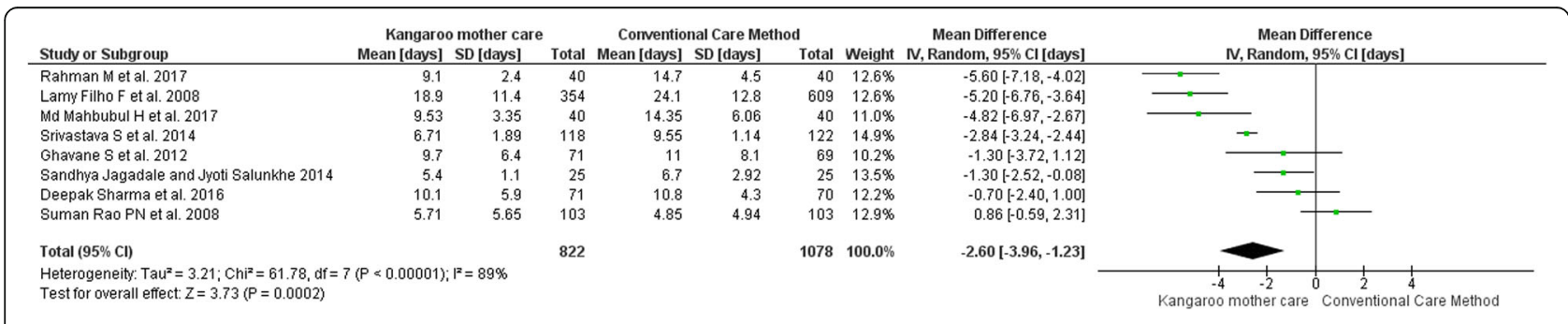

Fig. 3 Forest plot of pooled mean time difference to initiate exclusive breastfeeding among preterm and LBW infants 


\section{Discussion}

A systematic review of randomized controlled trials that comparing $\mathrm{KMC}$ and conventional neonatal care found compelling evidences which support KMC could improve breastfeeding rates in high-income countries in which conventional neonatal care is unavailable [10, 23]. In this meta-analysis, preterm and low birthweight infants in the KMC intervention group initiated breastfeeding 2.6 days earlier than conventional care method. This is in line with the previous systematic review studies in which KMC group initiated breastfeeding 1.6 days earlier than conventional neonatal care [10]. It was observed that infants who exposed to KMC showed significantly better emotion regulation than infants who exposed to the usual standard care which again initiated early breastfeeding [24, 25]. Furthermore, KMC has a significant role in starting breastfeeding among preterm and LBW infants [26-28]. Sloan NL et al. also reported that women in the community kangaroo mother care group initiated to breastfeed earlier than the control group [29]. This confirms the conclusion that KMC promotes early initiation of breastfeeding as compared to conventional care methods.

In this meta-analysis, preterm and LBW infants, initiated breastfeeding earlier than the conventional care group. Similarly, Moore ER et al. in their systematic review of randomized controlled studies, concluded that KMC improved the likelihood of breastfeeding initiation [30]. Various studies have also reported higher breastfeeding rates among KMC group as compared with conventional care method [28, 31, 32]. This could be explained by kangaroo mother care having a better effect on thermal regulation and reduced levels of infant stress, and is more comfortable than conventional neonatal care.

\section{Limitations}

In this analysis, the included studies were limited in number (only eight studies) which did not enable us to determine an effect size on all outcomes. The study searched only English language reports. In addition, this study was based only on published peer-reviewed studies and important data might be missed from unpublished studies.

\section{Conclusions}

Women in the kangaroo mother care intervention group, initiated breastfeeding earlier than conventional care method. Kangaroo mother care promotes early initiation of breastfeeding as compared to conventional care method. Therefore, health facilities need to implement the kangaroo mother care for preterm and low birthweight infants.

\section{Additional file}

Additional file 1: MEDLINE via PubMed and Cochrane database search for randomized controlled trial studies of $\mathrm{KMC}$ versus conventional care method, June 30, 2018. (DOCX 150 kb)

\section{Abbreviations \\ KMC: Kangaroo mother care; LBW: Low birthweight; RCT: Randomized controlled trial}

\section{Acknowledgments}

Not applicable.

\section{Funding}

The authors did not receive any funding from any organization.

\section{Availability of data and materials}

The datasets used and/or analyzed during the current study are available from the corresponding author on reasonable request.

\section{Authors' contributions}

AG, SS, and AD conceived and designed the study. All authors identified the study, extract the data, analyzed and interpreted the data and wrote the manuscript. All authors read and approved the final version of the manuscript.

Ethics approval and consent to participate

Not applicable.

Consent for publication

Not applicable.

\section{Competing interests}

The authors declared that they have no any competing interests.

\section{Publisher's Note}

Springer Nature remains neutral with regard to jurisdictional claims in published maps and institutional affiliations.

Received: 2 August 2018 Accepted: 13 February 2019

Published online: 19 February 2019

\section{References}

1. Charpak N, Ruiz-Peláez JG, Charpak Y. Rey-martinez kangaroo mother program: an alternative way of caring for low birth weight infants? One year mortality in a two cohort study. Pediatrics. 1994:94(6):804-10.

2. Tharashree CD, Shravani MR, Srinivasa S. The effect of kangaroo mother care (KMC) on breast feeding at the time of NICU discharge. Int J Contemp Pediatr. 2018;5(3):1068-71.

3. Charpak N, Ruiz-Peláez JG, De Calume Figueroa Z. Current knowledge of kangaroo mother intervention. Curr Opin Pediatr. 1996;8(2):108-12.

4. Dehghani K, Movahed Z, Dehghani H, Nasiriani K. A randomized controlled trial of kangaroo mother care versus conventional method on vital signs and arterial oxygen saturation rate in newborns who were hospitalized in neonatal intensive care unit. J Clin Neonatol. 2015:4:26-31.

5. Sharma A. Efficacy of early skin-to-skin contact on the rate of exclusive breastfeeding in term neonates: a randomized controlled trial. African Health Hciences. 2016;16(3):790-7.

6. Sampaio ÁR, Bousquat A, Barros C. Skin-to-skin contact at birth: a challenge for promoting breastfeeding in a "baby friendly" public maternity hospital in Northeast Brazil. Epidemiologia e Serviços de Saúde. 2016;25(2):281-90.

7. Elhalik M, El-Atawi K. Breast feeding and kangaroo care. J Pediatr Neonatal Care. 2016;4(6):00160.

8. Feldman $\mathrm{R}$, Eidelman Al, Sirota $\mathrm{L}$, Weller A. Comparison of skin-to-skin (kangaroo) and traditional care: parenting outcomes and preterm infant development. Pediatrics. 2002;110(1):16-26.

9. Badiee Z, Faramarzi S, MiriZadeh T. The effect of kangaroo mother care on mental health of mothers with low birth weight infants. Adv Biomed Res. 2014;3. 
10. Conde-Agudelo A, Diaz-Rossello JL. Kangaroo mother care to reduce morbidity and mortality in low birthweight infants. Cochrane Database Syst Rev. 2016;8:CD002771

11. Mahmood I, Jamal M, Khan N. Effect of mother-infant early skin-to-skin contact on breastfeeding status: a randomized controlled trial. Journal of Coll Physicians Surg Pak. 2011;21(10):601-5.

12. Tully KP, Holditch-Davis D, White-Traut RC, David R, O'Shea TM, Geraldo V. A test of kangaroo care on preterm infant breastfeeding. J Obstet Gynecol Neonatal Nurs. 2016;45(1):45-61.

13. Quigley M, McGuire W. Formula versus donor breast milk for feeding preterm or low birth weight infants. Cochrane Database Syst Rev. 2014;4: CD002971.

14. Charpak N, Ruiz-Peláez JG, Charpak Y. A randomized controlled trial of kangaroo mother care: results of follow-up at 1 year of corrected age. Pediatrics. 2001:108(5):1072-9.

15. Jagadale S, Salunkhe J. To evaluate the effectiveness of kangaroo mother care on low birth weight babies. Int J Sci Res. 2017;3(8):2319.

16. Lamy Filho F, Silva AA, Lamy ZC, Gomes MA, Moreira ME. Evaluation of the neonatal outcomes of the kangaroo mother method in Brazil. J Pediatr. 2008;84(5):428-35.

17. Hoque MM, Jahan N, Rahman MM, Saha LC, Akhter RJ, Chowdhury MA. Effectiveness of kmc on success of breast feeding in preterm low birth weight neonate. Academic J Pediatr Neonatol. 2017;3(4):555617.

18. Rahman M, Chowdhury M, Hoque M, Jahan N, Shaha LC. Kangaroo mother care for low birth weight babies: a randomized controlled trial in a tertiary care hospital of Bangladesh. J Pediatr Neonat Care. 2017:7(2):00285.

19. Srivastava S, Gupta A, Bhatnagar A, Dutta S. Effect of very early skin to skin contact on success at breastfeeding and preventing early hypothermia in neonates. Indian J Public Health. 2014;58(1):22.

20. Sharma D, Murki S, Pratap OT. The effect of kangaroo ward care in comparison with intermediate intensive care on the growth velocity in preterm infant with birth weight< $1100 \mathrm{~g}$ : randomized control trial. Eur J Pediatr. 2016:175(10):1317-24.

21. Ghavane S, Murki S, Subramanian S, Gaddam P, Kandraju H, Thumalla S. Kangaroo mother care in kangaroo ward for improving the growth and breastfeeding outcomes when reaching term gestational age in very low birth weight infants. Acta Paediatr. 2012:101(12):e545-9.

22. Suman Rao PN, Udani R, Nanavati R. Kangaroo mother care for low birth weight infants: a randomized controlled trial. Indian Pediatr. 2008:45(1):17.

23. Venancio SI, Almeida HD. Kangaroo mother care: scientific evidences and impact on breastfeeding. J Pediatr. 2004;80(5):173-80.

24. Akbari E, Binnoon-Erez N, Rodrigues M, Ricci A, Schneider J, Madigan S, et al. Kangaroo mother care and infant biopsychosocial outcomes in the first year: a meta-analysis. Early Hum Dev. 2018;122:22-31.

25. Shrivastava SR, Shrivastava PS, Ramasamy J. Utility of kangaroo mother care in preterm and low birthweight infants. S Afr Fam Pract. 2013;55(4):340-4.

26. Akhtar $\mathrm{K}$, Haque M, Khatoon S. Kangaroo mother care: a simple method to care for low-birth-weight infants in developing countries. J Shaheed Suhrawardy Med College. 2013;5(1):49-54

27. Jayaraman D, Mukhopadhyay K, Bhalla AK, Dhaliwal LK. Randomized controlled trial on effect of intermittent early versus late kangaroo mother care on human milk feeding in low-birth-weight neonates. J Hum Lact. 2017;33(3):533-9.

28. Debes AK, Kohli A, Walker N, Edmond K, Mullany LC. Time to initiation of breastfeeding and neonatal mortality and morbidity: a systematic review. BMC Public Health. 2013;13(suppl 3):19.

29. Sloan NL, Ahmed S, Mitra SN, Choudhury N, Chowdhury M, Rob U, et al. Community-based kangaroo mother care to prevent neonatal and infant mortality: a randomized controlled cluster trial. Pediatrics. 2008;121(5): e1047-59.

30. Moore ER, Bergman N, Anderson GC, Medley N. Early skin-to-skin contact for mothers and their healthy newborn infants. Cochrane Libr. 2016;11: CD003519.

31. Hake-Brooks S, Anderson GC. Kangaroo care and breastfeeding of motherpreterm infant dyads 0-18 months: a randomized controlled trial. Neonatal Network. 2008;27(3):151-9.

32. Mörelius $E$, Angelhoff $C$, Eriksson J, Olhager E. Time of initiation of skin-toskin contact in extremely preterm infants in Sweden. Acta Paediatr. 2012; 101(1):14-8

Ready to submit your research? Choose BMC and benefit from:

- fast, convenient online submission

- thorough peer review by experienced researchers in your field

- rapid publication on acceptance

- support for research data, including large and complex data types

- gold Open Access which fosters wider collaboration and increased citations

- maximum visibility for your research: over $100 \mathrm{M}$ website views per year

At BMC, research is always in progress.

Learn more biomedcentral.com/submissions 